\title{
Biosensor Technology in Diagnostics and Mechanistic Studies of Epilepsy ${ }^{\dagger}$
}

\author{
Michael Thompson * and Amanda Ackroyd \\ Department of Chemistry, University of Toronto, Toronto, ON M5S, Canada; a.ackroyd@mail.utoronto.ca \\ * Correspondence: m.thompson@utoronto.ca; Tel.: +1-416-978-3575 \\ + Presented at the 1st International Electronic Conference on Biosensors, 2-17 November 2020; Available \\ online: https://iecb2020.sciforum.net/.
}

Received: date; Accepted: date; Published: date

\begin{abstract}
Epilepsy is represented by a set of neurological disorders that result in recurring seizures and convulsions. Although several types of the condition have been characterized, the underlying cause for these remains largely unknown. A number of molecular biomarkers for epilepsy have been identified including glutamate, $\gamma$-aminobutyric acid and miRNAs. In addition, a special role appears to be played by the potassium cation. Detection of these species is anticipated to assist in both diagnosis and fundamental understanding of the condition. This review details the application of a number of biosensor devices that have been designed specifically for the detection of both molecular biomarkers and the $\mathrm{K}^{+}$cation in proximity to an animal cortex. These devices offer considerable potential not only for diagnostic goals, but also for study of the cause and spread of the epileptic seizure, especially if such biosensors can detect analytes in a multiplexed, real-time manner.
\end{abstract}

Keywords: epilepsy; molecular biomarkers; glutamate; GABA; miRNAs; potassium cation; relevant biosensors

\section{Epilepsy}

Epilepsy is associated with a set of neurological disorders that result in seizures [1-3]. The condition is termed the ictal state, where the most prevalent type of seizure is convulsive in character. In epilepsy, there is chronic aberrant neuronal and glial activity, however, seizures may also result from injury, changes in blood glucose levels, congenital heart disorders, or even be psychological in cause [4]. According to the World Health Organization (WHO), epilepsy affects over 65 million people worldwide [5]. Tragically, the condition results in over 100,000 deaths annually. Nearly $30 \%$ of patients with access to appropriate healthcare are not alleviated from their symptoms with currently available medications (e.g., anticonvulsants). This is because the abnormal neuronal firing and pathological changes may arise from multiple pathways, and treatment is unclear. Excitability may be from neurodegeneration or neurogenesis, abnormal synaptic connectivity, blood-brain barrier damage, or genetic channelopathies (mutations in ion-channels or the $\gamma$-aminobutyric acid A receptor $\left(\mathrm{GABA}_{A} \mathrm{R}\right)$ [6]. The precise mechanism that underlies epilepsy is unknown, although research has revealed a number of factors concerning cellular processes. However, it is unclear what instigates the brain to proceed to excessive synchronization of neuronal activity, a key feature of the seizure.

The diagnosis of epilepsy is typically complex requiring the application of several assays and techniques [7]. Complications also arise from the fact that other medical conditions, such as migraines and narcolepsy, exhibit similar symptoms to those that occur in epilepsy. Unfortunately, missed diagnosis occurs in up to $30 \%$ of cases. Generically, blood tests are performed on an initial basis to 
rule out issues associated with the concentration of glucose and other species and the presence of infection. Several electroencephalograms (EEG) may be recorded in order to observe the electrical activity of the brain in different environmental settings, for lengths of time, or with different arrangements of electrodes on the scalp. Structural damage or abnormalities in the brain (if present) are then imaged with computerized tomography (CT) scans or magnetic resonance imaging (MRI).

Aside from conventional blood assays in epileptic diagnosis, the identification of molecular and cellular biomarkers is a critical component of research into the causes, mechanisms and potential prevention and treatment of the condition by pharmaceutical intervention [6,8]. (A biomarker is a quantifiable species whose presence is indicative of the occurrence of a medical condition such as disease.) In this regard, the statement by Carlen [9] that "epilepsy is not an electrographic disease, but rather a complex manifestation of underlying biochemical and biophysical abnormalities associated with electrographic abnormalities which reflect as well as possibly contribute to the underlying brain dysfunction" is especially relevant and intriguing. Biosensor technology offers unique potential for both epileptic diagnostics and fundamental research in that such devices can provide rapid detection of relevant biochemical concentrations in a potentially multiplexed approach. In this regard, a concise look at selected molecular (and ionic) biomarkers relevant to sensor development, as distinct from imaging markers [6,8], is outlined in the next section.

\section{Biomarkers for Epilepsy}

\subsection{Molecular Species}

Glutamate (Glu), which is an important neurotransmitter, has long been recognized as having a very significant role in the instigation and excitatory activity of epileptic seizure [10]. Unsurprisingly, this observation has spawned numerous studies over the years. There is an apparent increase in extracellular concentration prior to the onset of seizures and indeed during the spread of the condition. The neurochemistry of Glu including the various receptors involved in the synapse is exceedingly complex and at the present time not well understood [11]. There has been significant effort to design drugs which can ameliorate issues with respect to Glu but deleterious side effects appear to present difficulties in treating seizures.

The neurotransmitter, $\gamma$-Aminobutyric acid (GABA), is also a major player in the cerebral cortex [12]. It is the main inhibitory neurotransmitter in the cerebral cortex producing synchronization of activity in cortical networks. The function of the GABAercic system is designed to compensate for the activity of the aforementioned excitatory glutamatergic process. Research has shown that masking of the GABAergic system can result in seizures. As for glutamate, considerable attention has been placed on drugs that influence GABA receptors in order to attempt treatment of seizure activity.

A relatively recent advance in the consideration of potential molecular biomarkers for epilepsy is the role played by micro RNA species (miRNAs) [13]. These molecules are non-coding RNAs that function to control protein levels in all cells. It has been shown that levels of a number of brainenriched miRNAs are clearly altered in patients suffering from epilepsy. Very relevant to the issue of epilepsy characterization are the observations that the several miRNAs are stable in biofluid and can be detected by standard PCR technology. Despite this promise it is the case that much remains to be researched with regard to the precise connection between the mechanisms that lie behind epilepsy and the brain presence of specific miRNAs.

\subsection{The Critical Role of Potassium Cation}

Considerable research has been directed at the relationship between potassium cation concentration and the presence of a number of neurological disorders, in particular with regard to the cerebrospinal fluid (CSF) that bathes the brain [14]. An example is the research of Uva et al. [15] on the connection between seizures and extra-cellular potassium concentration. In this work, $\mathrm{K}^{+}$sensitive double-barrel electrodes were placed near the pial area (olfactory cortex), i.e., the surface between grey matter and CSF, for correlation of the extracellular concentration of the cation with 
seizure-like events. In recent years, particular interest has been shown in neuronal potassium channels, especially the effect that alterations by mutation and malfunctions of a variety of such channels have on epileptic seizures [16]. As mentioned above, these effects are referred to in terms of " $\mathrm{K}^{+}$channelepsies", where there is an applied link between channel abnormalities and the epileptic condition [17]. In the latter publication, the authors detail carefully the characteristics of potassium dynamics during epileptic events. They conclude that "it is now well-recognized that enhanced (concentration) of extra-cellular potassium is both a consequence of seizure activity and the cause of brain tissue hyperexcitability". In reality, at the present time it is unclear if seizures are instigated by abnormalities in extracellular potassium, whatever the cause of that phenomenon, or a consequence of the condition, or both these processes.

\section{Relevant Biosensor Development}

Given the observation that a number of biomarkers [6.8,13] have been identified in terms of association with epileptic seizures it is surprising that measurement by biosensor has not attracted significant attention, as reflected in the literature. Indeed, there has, unsurprisingly, been heavy emphasis on the detection of glutamate. Here, we detail selected studies that have been performed, although it is fair to state that it is often the case that explicit measurements related to epilepsy are notably missing.

\subsection{Glutamate}

In 2014, B. Batra, et al. [18] published research on the fabrication of a device for the detection of Glu based on a coated gold electrode. The focus of this work was to detect glutamate in food, while pointing out the importance of the molecule in epilepsy. At the time of writing, the authors argued that their glutamate biosensor had the lowest published limit-of-detection (LOD). Glutamate oxidase (GOx) was covalently bound to a composite film of polypyrrole nanoparticles (PPyNPs) and polyaniline (PANI) on gold $(\mathrm{Au})$ electrodes through glutaraldehyde coupling. PANI provides an $\mathrm{NH}_{2}$ functional group to react with a $-\mathrm{CHO}$ on GOx. The reaction was confirmed by Fourier Transform infrared (FTIR) spectroscopy and X-ray diffraction (XRD). The amperometric biosensor determined the L-glutamate in the matrix with an optimum response at $\mathrm{pH} 7.5$ and $35^{\circ} \mathrm{C}$, which the authors argue is close to physiological conditions, despite this being a temperature at which hypothermia would occur. To measure the effectiveness of the biosensor, the Glu biosensor was tested in tomato, noodles, and tomato soup, but only under the aforementioned conditions. Samples were spiked, and at $5 \mathrm{mM}$, there was a $95.6 \%$ recovery.

C.A. Cordeiro, et al. [19] developed an implantable multiplex microbiosensor and claimed it would function well for studies of epilepsy. Figure 1 shows that the Glu/Lac/Pyr biosensor was made with a layer by layer method. The needle-type platinum micro-electrodes were first layered with a permselective poly(phenylenediamine) membrane to prevent unwanted molecules from diffusing to the electrode. Then, GOx, lactate oxidase (LOx) and pyruvate oxidase (POx) were mixed with hydrogels, and crosslinked with glutaraldehyde and bovine serum albumin (adsortion prevention).

In the in vivo trial, it took $30-45 \mathrm{~min}$ for a stable basal electro chemical to be reached. Brain Glu levels of a rat are $1.3 \mathrm{mM}$, Lac are $0.3-2 \mathrm{mM}$, and Pyr are 0.08 to $0.016 \mathrm{mM}$. The results were an average of $1.3 \mathrm{mM}$ for Glu, $1.56 \mathrm{mM}$ for Lac and $0.29 \mathrm{mM}$ for Pyr. The high report for Pyr is because the authors claim that they have better sensitivity than all previous research. In addtion, the claim was made that, although the biosensor dispalays significant biofouling, it reamis highly selective in terms of signal devlopment. Finally, the authors claim that this system has potential use for epileptic diagnosis, without actually performing any studies on animals to justify their claim. 


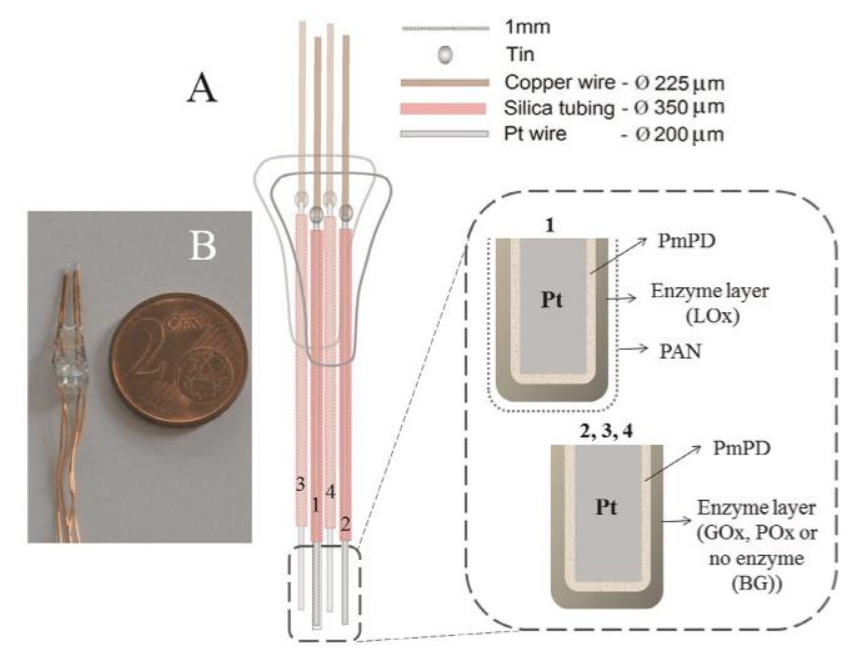

Figure 1. Schematic of (A) the biosensor probe with the (1) Lac, (2) Glu, (3) Pyr and (4) background biosensors; (B) 2 cent Euro coin. (Reprinted by kind permission of Elsevier).

Ganesana, et al. [20] claim to have produced an easily-fabricated and disposable novel Glu biosensor for in vivo monitoring. Figure 2 depicts a schematic of the Glu biosensor.

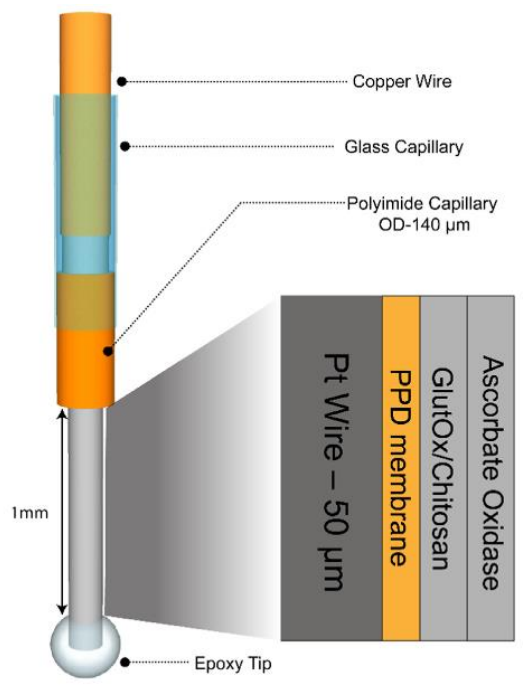

Figure 2. Schematic of the Glu biosensor; the Pt wire is only $50 \mu \mathrm{m}$ in diameter. (Reprinted by kind permission of Elsevier).

Poly-o-phenylenediamine (PPD) is used as a permselective membrane, GOx is trapped in a chitosan matrix, and is protected by ascorbate oxidase (AsOx) to prevent its release. \{Ascorbic acid is present in the central nervous system in 200-400 $\mu \mathrm{M}$ amounts. To avoid non-specific adsorption (NSA), the probe was dipped in BSA before use. To mimic in vitro measurements, the authors used a mixture of serotonin, adenosine, dopamine, glucose, uric acid, and ascorbic acid. In contrast to this experiment being conduted for $10 \mathrm{~min}$, the in vivo measurements were conducted over $4 \mathrm{~h}$. The biosensor was determined to have a linear range of glutamate from 5 to $150 \mu \mathrm{M}$, and a limit of detection of $0.044 \mu \mathrm{M}$. In the in vivo rat experiments, a stable baseline was achieved at $30 \mathrm{~min}$. Pulse stimulations caused an increase of glutamate subthalamic nucleus, which resulted in easily detectable micromolar changes in the range of 3-20 $\mu \mathrm{M}$.

A glutamate microbiosensor with ceramic-substrate enabled platinum microelectrode array (PtMEA) was made by drop casting GOx, BSA and glutaraldehyde, and subsequently $\mathrm{m}$ phenylenediamine after drying [21]. The magnitude of the current when $\mathrm{Pt}$ oxidizes $\mathrm{H}_{2} \mathrm{O}_{2}$ results in 
a measurement of the Glu concentration. This methodology is very similar to much of previously mentioned literature. However, the uniqueness of this paper is probing rat cell lines of (1) astrocytes, which are glial cells that help form the physical structure of the brain, and express glutamate transporters, and (2) glioma cells, which are a form of brain cancer. It is believed that impaired uptake of glutamate by astrocytes may result in seizures. The probe was able to measure 10-570 $\mu \mathrm{M}$ Glu in vitro, with a sensitivity of $62.3 \pm 6.1 \mathrm{nA} / \mu \mathrm{M} \mathrm{cm}{ }^{2}$ in basal media and $270 \pm 28 \mathrm{nA} / \mu \mathrm{M} \mathrm{cm}{ }^{2}$ in PBS buffer, which is nearly four times better than other Pt-MEA Glu biosensors.

Figure 3 shows the measurements made with consecutive spiking of basal media (4a), astrocytes (4b) and glioma cells (4c) with Glu. It is shown that astrocytes are able to return to baseline after Glu uptake, whereas basal media and glioma cells do not. With this data, the authors were able to differentiate between impaired Glu transport in glioma cells and normal transport in astrocytes.
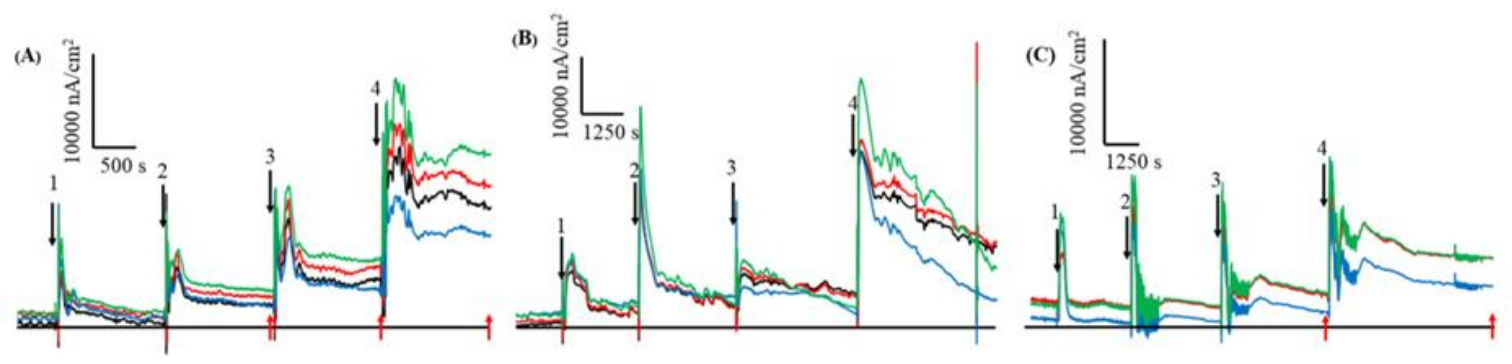

Figure 3. Real-time measurements of additions of Glu (red arrows at base) (A) shows no cells present, and that there is no return to baseline as Glu increases (B) Astrocyte traces show a return to baseline after addition of Glu indicating Glu uptake and (C) Glioma cells show increase in traces with no return to baseline at increased concentration. Glu levels at $1=10 \mu \mathrm{M}, 2=30 \mu \mathrm{M}, 3=70 \mu \mathrm{M}, 4=170 \mu \mathrm{M}$; variation in line colour refers to different electrodes. (Reprinted by kind permssion of Elsevier).

\section{2. $G A B A$}

As mentioned above, GABA is a major inhibitor neurotransmitter that helps with plasticity and network synchronization in the brain. To determine the continuous concentration of GABA, Hossain, et al. [22] developed a probe capable of detection at two sites. On the first a Pt electrode, only GOx is present, while for the second Pt electrode has both GOx and GABASE. Both were coated with glutaraldehyde and BSA, as well as m-phenylenediamine. The amount of GABA present was determined from the difference of the $\mathrm{H}_{2} \mathrm{O}_{2}$ current at each sensor:

$$
\mathrm{IGABA}_{\mathrm{G}}=\mathrm{IH}_{\mathrm{H}} \mathrm{O} \text { (Site 2) }- \text { IH2O2(Site 1) }
$$

What is unique about this biosensing device, is that no extra reagents are required to achieve a signal, since in situ $\alpha$-ketoglutarate is a product of glutamate oxidation. The key reaction will be continuous in vivo because glutamate is readily available. This biosensor was able to measure GABA with 26

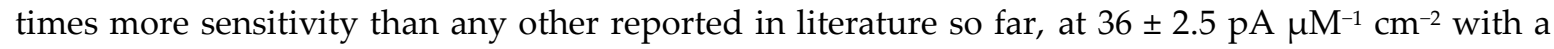
LOD of $2 \mu \mathrm{M}$ in vitro, while the Glu results are only comparable. In ex vivo experiments of the rat hippocampal brain slices, during stimulations of the rat brain, Glu concentrations ranged from 5 to $25 \mu \mathrm{m}$, and GABA ranged from 5 to $13 \mu \mathrm{M}$.

\section{3. $\operatorname{miRNAs}$}

As implied above, recent times have seen rapildy developing interest in miRNAs because of their potential utility in medical diagnostics. A number of approaches have been empoyed to detect these species such as Northern blotting, quantitative real-time PCR, microarray tecchnology and biosensor devices [23]. However, the focus of research has been very much centered on detection associated with various forms of cancer. There has been virtually no mention of the application of miRNA biosensors to research in epilepsy despite the importance of these species referred to above. Given this observation, we restrict this discussion to a couple of recent examples of systems that could 
be employed in the neurology field, although it is evident that some form of miniaturization would clearlry need to be invoked in the future.

A supposedly label-free aptamer-based biosensor was developed for miRNA assay by RNAregulated fluorescence of malachite green (MG) [24]. In this approach, target miRNA is hybridized with the DNA extension template to form the T7 in vitro transcription system. The resulting transcription amplification produced a large number of MG RNA aptamers (MGAs) which increase the fluorescence of the MG, achieving significant fluorescence enhancement for miRNA quantitative analysis. The aptamer-based biosensor is said to provide a low detection limit of 10 amol target miRNA. In this case the target analyte was miRNA connected to lumg cancer, although no actual samples were assessed. Lung cancer detection was also the recent focus of another fluorescencebased assay [25]. In order to detect miRNA-155 expression levels a modified La(III)-metal-organic framework(MOF) and silver nanoparticles (Ag NPs) were used as the energy donor-acceptor pairs in fluorescence quenching through the FRET process. The framework was modified by glutaraldehyde as a cross-linking agent. Additionally, Ag NPs were prepared, and then, the surface of both was conjugated with different $5^{\prime}$-amino-labeled ssDNA strands (aptamers). The system was capable of detecting $0.04 \mathrm{ppb}$ (ng. $\mathrm{mL}^{-1}$ ) or $5.5 \mathrm{fM}$ of the miRNA-155 biomarker. Again no appraisal of practical samples appeared to have been attempted.

Several other optical and electrochemical deices have aso been employed in order to detect niRNAs, examples being those based on graphene modified FETs [26] and localized surface plasmon resonance [27]. Again, the focus in these cases is largely on cancer detection.

\subsection{Potassium Cation}

One of the earliest measurements related to epilepsy was that of Heinemann et al. [28] who detected time responses for both $\mathrm{Ca}^{2+}$ and $\mathrm{K}^{+}$, using ion selective electrodes with 2 micron tips, during seizures instigated in the cortex of an animal. The concentration of the calcium ion decreased in contrast to potassium which increased, an important early observation.

Micro-electrodes were used to determine both extracellular calcium and potassium concentrations in the cerebral cortex of cats during patterns of slow sleep oscillations and spike-wave seizures [29]. Results obtained during the slow oscillation indicated that glial cells phasically take up part of the extracellular $\mathrm{K}^{+}$extruded by neurons during the depolarizing phase of the slow oscillation.

During this condition, the redistribution of potassium appeared to be local. Large steady increases in $\mathrm{K}^{+}$and phasic potassium accumulations were measured during spike-wave seizures.

In particularly ground-breaking experiments, Dufour et al. [30] employed a biosensor system consisting of a potassium micro-electrode for extracellular assays paired with both a second microelectrode for field potential determinations and a tapered fiber optic device for intracellular assay of the cation (Figure 4). The performance of the optrode configuration was assessed via experiments involving animal sleep-like patterns, induced seizures (with penicillin), and anaesthesia comatose states. It was observed that paroxysmal discharges were associated with positive variations of the intracellular fluorescence corresponding to extracellular $\mathrm{K}^{+}$increases. In the other cases, intracellular $\mathrm{K}^{+}$was reduced for an extracellular $\mathrm{K}^{+}$increase. Comatose states were accompanied by an extracellular drop of $\mathrm{K}^{+}$of $1.31 \pm 0.13 \mathrm{mM}$, which was reflected, in all cases, by an intracellular $\mathrm{K}^{+}$ increase of $39 \pm 4 \%$. 


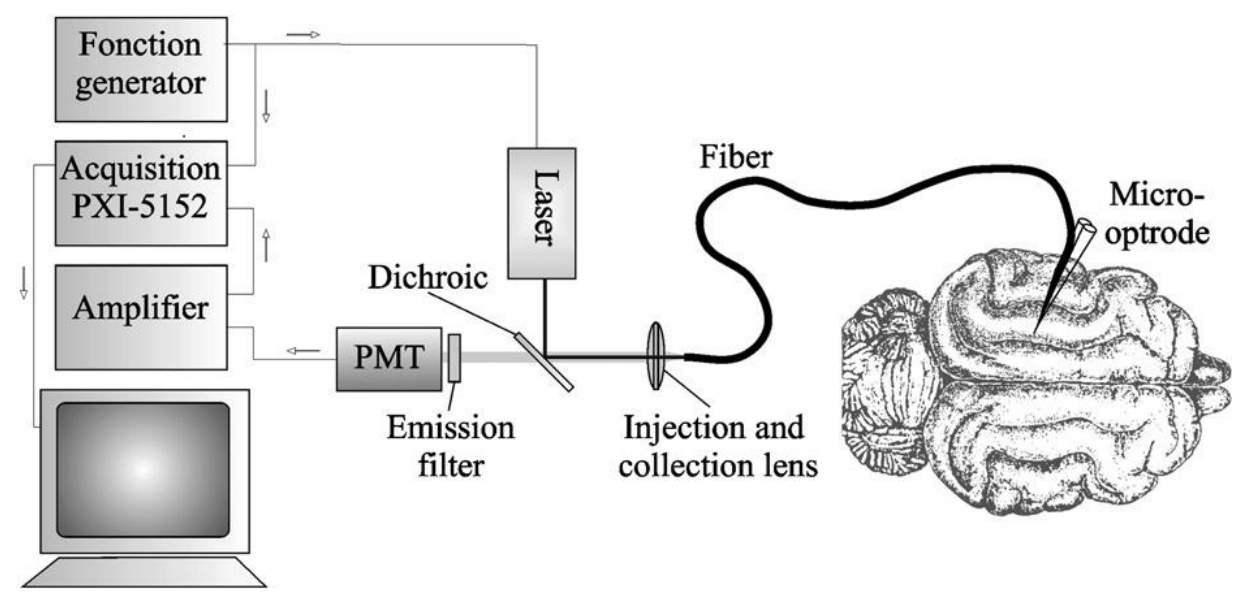

Figure 4. Excitation light generated by the laser is reflected by a dichroic filter and directed through an aspheric injection lens toward the multimode delay fiber and further to the micro-optrode. After excitation of the tissue, fluorescence is collected with the same optical fiber, re-collimated by the aspheric lens, directed through the dichroic mirror, filtered with a $40 \mathrm{~nm}$ large band pass filter cantered on $500 \mathrm{~nm}$, to finally reach a photomultiplier tube (PMT) detector. The PMT signal is amplified and digitized. (Reprinted by kind permission of Elsevier.).

In our own research we have developed a gold-based micro-electrode array (six sensors) in order to perform spatio-temporal mesurements of potassium cation in mouse brain [31]. A newlysynthesized electrode coating for potassium binding, coupled with antifouling surface chemistry was employed for selective detection of the cation (Figure 5). The configuration included both reference (2) and working (4) electrodes, which were interrogated by impedance spectroscopy via a sweep from $100 \mathrm{~Hz}$ to $1 \mathrm{kHz}$ obtained in real time. Figure 6 shows the results for $\mathrm{K}^{+}$calibraton and included comparison with standard electrodes.

Two types of in vivo research were performed on the the isolated cerebral cortex of an anaesthetized mouse. In the first experiment, optogenetic stimulation was used to instigate extracellular potassium concentration rises. Secondly, transient potassium accumulation was also recorded after accompanying seizure-like events were evoked through the application of a 4aminopyridine solution onto the exposed cortical surface. Optogenetic stimulation of the mouse cortex caused concentrations of $\mathrm{K}^{+}$that decreased transiently at the onset of stimulation, followed by a sharp increase of $2 \mathrm{mM}$ for a period of less than $100 \mathrm{~ms}$. Return to equilibrium at $4 \mathrm{mM}$ was observed immediately for each subsequent stimulation event. For chemically-induced epileptic seizures, an increase in concentration in $\mathrm{K}^{+}$of approximately $10 \mathrm{mM}$ for each channel was observed, although the rise of the cation in one channel lagged behind those of the other two channels. This spatio-temporal specificity may indicate that epileptic seizures occur in an entire region of the brain, rather than at a very specific starting point. 


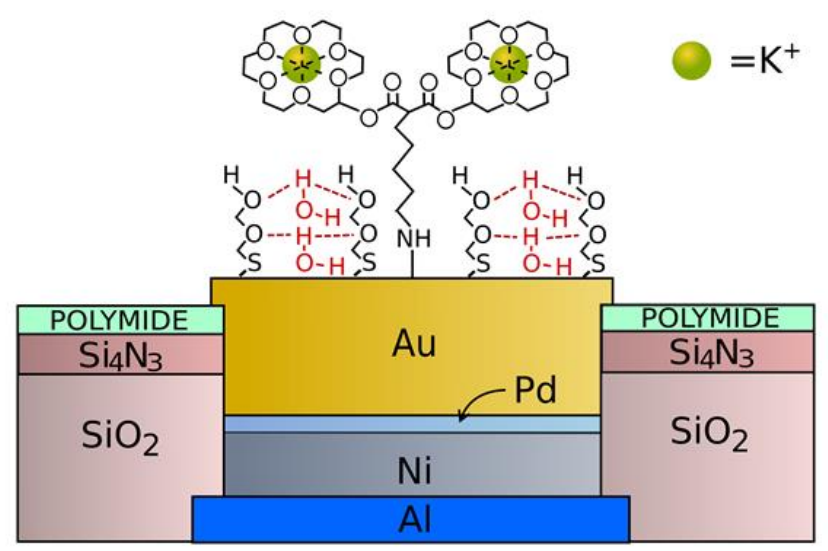

Figure 5. Electrode structure with $\mathrm{K}^{+}$sensitive layer. The cown ether probe is embedded in a selfassembled monolayer (SAM) to effect antifouling capability. The ratio of probe-to-SAM is designed to maximize the impedimetric signal while reducing fouling from CSF components.
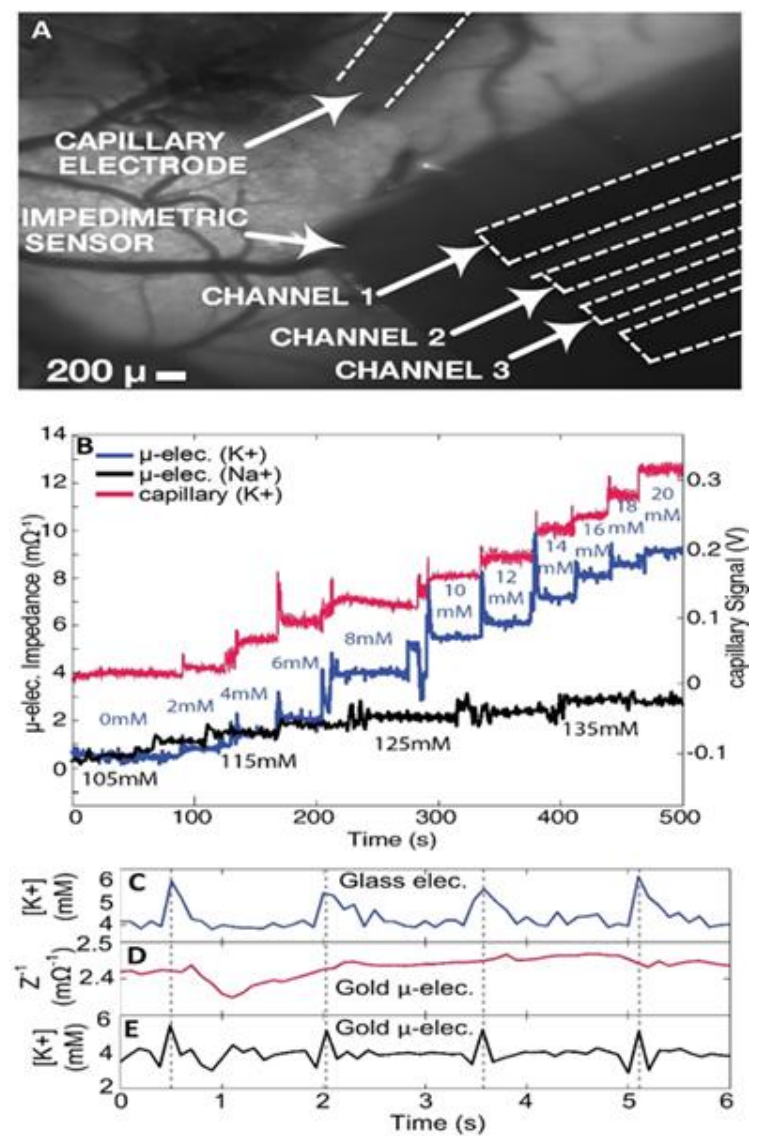

Figure 6. (A) Raw fluorescence image of a mouse cranial window, with $10 \times$ magnification: the glass capillary electrode (top) and monolayer gold-coated electrode (right), (B) calibration curves of both the glass capillary electrode (red) and coated gold micro-electrode (blue) for varying concentratons of $\mathrm{KCl}$ and $\mathrm{NaCl}$. (C-E) In vivo recordings of extracellular potassium during optogenetically-elicited responses for both glass capillary (blue) and coated gold micro-electrodes (black). 


\section{Final Comments}

Biosensor technology offers distinct possibilities to contribute to diagnostic assays, fundamental studies of the epileptic condition, and the development of antiepileptogenic and antiseizure drugs. In particular, animal modeling with such devices will allow the prediction of the condition, assay its progression and potentially provide a convenient platform for drug screening. One of the great strengths of the technology will be the capability to generate multiplexed signals for the presence and concentration of a number of biomarker targets in the CSF simulataneously, and in real time. Obviously, it will be mandatory to employ micro- or nano-devices for such a purpose. An excellent example of this approach is the potential offered by electrochemical detection via the multi-electrode microarray [32]. A key issue, however, which has represented something of an "Achilles Heel" for biosensor technology, is the fouling of the device surface when placed in biological fluids such as CSF. Fortunately, possible solutions to this problem have been the subject of significant research attention in recent years [33].

In the future, it may be feasible to fabricate a wireless, wearable system for interrogating the brain such as that decsribed Sawan et al. [34]. In this case, the approach was the measurement of simultaneous near-infrared spectrometry (NIRS) and scalp electro-encephalography (EEG) for noninvasive monitoring and intracerebral EEG (icEEG) for invasive monitoring. Although this system was not configured for analyte detection, the introduction of such measurents in tandem with those from electrophysiology may well represent a particularly excitimg direction in the study of the epileptic condition.

Author Contributions: Writing - original draft, A.A.; Writing and editing, M.T.

Acknowledgments: The authors are deeply appreciative of the enthusiastic support and very helpful discussion provided by Peter Carlen of the Krembil Research Institute, Toronto Western Hospital, Toronto.

Conflicts of Interest: The authors declare no conflict of interest.

\section{References}

1. Chang, B.S.; Lowenstein, D.H. Epilepsy. N. Engl. J. Med. 2003, 349, 1257-1266.

2. Fisher, R.S.; Boas, W.V.E.; Blume, W.; Elger, C.; Genton, P.; Lee, P.; Engel, J., Jr. Epileptic seizures and epilepsy: definitions proposed by the International League Against Epilepsy (ILAE) and the International Bureau for Epilepsy (IBE). Epilepsia 2005, 46, 470-472.

3. Alarcón, G.; Valentin, A. (Eds.) Introduction to Epilepsy; Cambridge University Press: Cambridge, UK, 2012.

4. Reuber, M.; Elger, C.E. Psychogenic nonepileptic seizures: Review and update. Epilepsy Behav. 2003, 4, 205216.

5. Dumanis, S.B.; French, J.A.; Bernard, C.; Worrell, G.A.; Fureman, B.E. Seizure forecasting from idea to reality. Outcomes of my seizure gauge epilepsy innovation institute workshop. eNeuro 2017, 4 .

6. Pitkänen, A.; Ndode-Ekane, X.E.; Lapinlampi, N.; Puhakka, N. Epilepsy biomarkers-Toward etiology and pathology specificity. Neurobiol. Dis. 2019, 123, 42-58.

7. Angus-Leppan, H. Diagnosing epilepsy in neurology clinics: A prospective study. Seizure 2008, 17, 431436.

8. Engel, J., Jr.; Pitkänen, A.; Loeb, J.A.; Dudek, F.E.; Bertram, E.H., III; Cole, A.J.; Moshé, S.L.; Wiebe S.; Fureman, B.E.; Jensen, F.E.; et al. Epilepsy Biomarkers. Epilepsia 2013, 54, 61-69.

9. Carlen, P.L. Krembil Research Institute, Toronto Western Hospital, Toronto, Ontario, Canada. Personal Communication.

10. Meldrum, B.S. The role of glutamate in epilepsy and other CNS disorders. Neurology 1994, 44 (Suppl. 8), S14-S23.

11. Barker-Haliski, M.; White, H.S. Glutamatergic mechanisms associated with seizures and epilepsy. Cold Spring Harb. Perspect. Med. 2015, 5, a022863.

12. Khazipov, R. GABAergic Synchronization in Epilepsy. Cold Spring Harb. Perspect. Med. 2016, 6, a022764.

13. Simonato, N.E.M.; Henshall, D.C. Discovery and validation of blood microRNAs as molecular biomarkers of epilepsy: Ways to close current knowledge gaps. Epilepsia Open 2018, 3, 427-436. 
14. Thompson, M. Neurophysiological monitoring of potassium. In Compendium of In Vivo Monitoring in ReaTime Molecular Neuroscience; Wilson, G.S., Michael, A.C., Eds.; World Scientific: Singapore, 2019; Volume 3, pp. 293-323.

15. Uva, L.; Saccucci, S.; Chikhladzi, L.; Tassi, L.; Gnatkovsky, V.; Milesi, G.; Morbin, M.; de Curtis, M. A novel focal seizure pattern generated in superficial layers of the olfactory cortex. J. Neurosci. 2017, 37, 3544-3554.

16. Villa, C.; Combi, R. Potassium channels and human epileptic phenotypes: An updated overview. Front. Cell Neurosci. 2016, 10, 81, doi: 10.3389/fncel.2016.00081.

17. De Curtis, M.; Uva, L.; Gnatkovsky, V.; Labrizza, L. Potassium dynamics and seizures: Why is potassium ictogenic? Epilepsy Res. 2018, 143, 50-59.

18. Batra, B.; Kumari SPundir, C.S. Construction of glutamate biosensor based on covalent immobilization of glutamate oxidase on polypyrrole nanoparticles/polyaniline modified gold electrode. Enzym. Microbial. Technol. 2014, 57, 69-77.

19. Cordeiro, C.A.; de Vries, M.G.; Ngabi, W.; Oonen, P.E.; Cremers, T.I.F.H.; Westerink, B.H.C. In vivo continuous and simultaneous monitoring of brain energy substrates with a multiplex amperometric enzyme-based biosensor device. Biosens. Bioelectron. 2015, 67, 677-686.

20. Ganesana, M.; Trikantzopoulos, E.; Maniar, Y.; Lee, S.T.; Venton, B.J. Development of a novel micro biosensor for in vivo monitoring of glutamate release in the brain. Biosens. Bioelectron. 2019, 130, 103-109.

21. Scoggin, J.L.; Tan, C.; Nguyen, N.H.; Kansakar, U.; Madadi, M.; Siddiqui, S.; Arumugam, P.U.; DeCosta, M.A.; Murray, T.A. An enzyme-based electrochemical biosensor probe with sensitivity to detect astrocytic versus glioma uptake of glutamate in real time in vitro. Biosens. Bioelectron. 2019, 126, 751-757.

22. Hossain, I.; Tan, C.; Doughty, P.T.; Dutta, G.; Murray, T.A.; Siddiqui, S.; Arumugam, P.U. A novel microbiosensor microarray for continuous ex vivo monitoring of gamma-aminobutyric acid in real-time. Front. Neurosci. 2018, 12, 500, doi:10.3389/fnins.2018.00500.

23. Kilic, T.; Erdem, A.; Ozsoz, M.; Carrara, S. microRNA biosensors: Opportunities and challenges among conventional and commercially available techniques. Biosens. Bioelectron. 2018, 99, 525-546.

24. Wang, H.; Wang, H.; Zhang, M.; Jia, Y.; Li, Z. A label-free aptamer-based biosensor for microRNA detection by the RNA-regulated fluorescence of malachite green. RSC Adv. 2019, 9, 32906-32910.

25. Afzalinia, A.; Mirzaee, M. Ultrasensitive fluorescent miRNA biosensor based on a "sandwich" oligonucleotide hybridization and fluorescence resonance energy transfer process using an $\operatorname{Ln}(\mathrm{III})-\mathrm{MOF}$ and Ag nanoparticles for early cancer diagnosis: Application of central composite design. ACS Appl. Mater. Interfaces 2020, 12, 16076-16087.

26. Gao, Y.; Han, Y.; Pang, J.; Wang, C.; Wang, Y.; Liu, H.; Zhang, Y.; Han, L. Ultrasensitive label-free miRNA sensing based on a flexible graphene field-effect transistor without functionalization. ACS Appl. Electron. Mater. 2020, 2, 1090-1098.

27. Thamm, S.; Müller, P.; Csáki, A.; Fritzsche, W.; Zuccheri, G. A miRNA biosensor based on localized surface plasmon resonance enhanced by surface-bound hybridization chain reaction, Biosens. Bioelectron. 2020, 167, 112465.

28. Heinemann, U.; HDLux, H.D.; Gutnick, M.J. Extracellular free calcium and potassium during paroxysmal activity in the cerebral cortex of the cat. Exp. Brain Res. 1977, 27, 237-243.

29. Amzica, F.; Massimino, M.; Manfridi, A. Spatial buffering during slow and paroxysmal sleep oscillations in cortical networks of glial cells in vivo. J. Neurosci. 2002, 22, 1042-1053.

30. Dufour, S.; Dufour, P.; Chever, O.; Vallée, R.; Amzica, F. In vivo simultaneous intra- and extracellular potassium recordings using a micro-optrode. J. Neurosci. Methods 2011, 194, 206-217.

31. Machado, R.; Soltani, N.; Dufour, S.; Salam, M.T.; Carlen, P.L.; Genov, R.; Thompson, M. Antifouling impedimetric sensor for the array high-resolution extracellular potassium monitoring in the brain. Biosensors 2016, 6, 53-64.

32. Gabran, S.R.I.; Salam, M.T.; Dian, J.; El-Hayek, Y.; Perez Velazquez, J.L.; Genov, R.; Carlen, P.L.; Salama, M.M.A.; Mansour, R.R. High-density intracortical microelectrode arrays with multiple metallization layers for fine-resolution neuromonitoring and neurostimulation. IEEE Trans. Neural Syst. Rehab. Eng. 2013, 21, 869-879.

33. Blaszykowski, C.; Sheikh, S.; Thompson, M. A Survey of state-of-the-art surface chemistries to minimize fouling from human and animal biofluids. Biomater. Sci. 2015, 3, 1335-1370. 
34. Sawan, M.; Salam, M.T.; Le Lan, J.; Kassab, A.; Gélinas, S.; Vannasing, P.; Lesage, F.; Lassonde, M.; Nguyen, D.K. Wireless recording systems: From noninvasive EEG-NIRS to invasive EEG devices. IEEE Trans. Biomed. Circuits Syst. 2013, 7, 186-195.

Publisher's Note: MDPI stays neutral with regard to jurisdictional claims in published maps and institutional affiliations.

(C) 2020 by the authors. Licensee MDPI, Basel, Switzerland. This article is an open access article distributed under the terms and conditions of the Creative Commons Attribution (CC BY) license (http://creativecommons.org/licenses/by/4.0/). 